\title{
PRINCIPAL ELEMENTS OF LATTICES OF IDEALS ${ }^{1}$
}

\author{
P. J. McCARTHY
}

AbSTRACT. The notion of principal element of a commutative multiplicative lattice was introduced by Dilworth. In this note the principal elements of the lattice of ideals of a commutative ring with unity $R$ are characterized as those ideals of $R$ which are finitely generated and locally principal ideals. It follows that a regular ideal of $R$ is a principal element of the lattice of ideals of $R$ if and only if it is invertible.

In [3], Dilworth introduced the notion of principal element of a commutative multiplicative lattice $L$ : an element $M$ of $L$ is a principal element of $L$ if

$$
(A \cap B: M) M=A M \cap B \text { and }(A \cup B M): M=A: M \cup B
$$

for all elements $A$ and $B$ of $L$. Dilworth showed that a principal ideal of a commutative ring with unity $R$ is a principal element of the lattice $L(R)$ of ideals of $R$, and he extended the Krull principal ideal theorem to principal elements of a Noether lattice [3, Theorem 6.4].

Throughout this note, let $R$ be a commutative ring with unity. It is natural to ask which ideals of $R$ are principal elements of $L(R)$. Dilworth [3, Theorem 7.1] showed that if $R$ is a unique factorization domain, then every principal element of $L(R)$ is a principal ideal of $R$. Bogart [1, Corollary 2.1] and Johnson [8, Corollary 1.5] showed that this is also the case when $R$ is a Noetherian local ring. On the other hand, as Dilworth pointed out, if $R$ is a Dedekind domain, then every ideal of $R$ is a principal element of $L(R)$. Janowitz [7] determined those rings which have this property; they are the Noetherian multiplication rings.

It is well known that an element of a Noether lattice is principal if and only if it is principal in every localization. This is true of every multiplicative lattice which has a suitable localization theory, i.e., for which the assertion of [9, Proposition 4] holds. Combining this with the result of Bogart and Johnson, we see that if $R$ is a Noetherian ring, then an ideal of $R$ is a principal element of $L(R)$ if and only if

Received by the editors September 15, 1970.

AMS 1970 subject classifications. Primary 13A15; Secondary 13 F05.

Key words and phrases. Principal element, invertible ideal.

1 This research was supported by University of Kansas Research Grant No. 3688-5038. 
it is a locally principal ideal. In this note we shall generalize this to rings which are not necessarily Noetherian.

It follows from our main result that the principal elements of $L(R)$ play the same role, in a sense, as the invertible ideals of $R$. In fact, it has been known for several years that if $R$ is an integral domain, then a nonzero ideal of $R$ is a principal element of $L(R)$ if and only if it is an invertible ideal of $R$. The necessity is clear. On the other hand, if $M$ is an invertible ideal of $R$ and if $B$ is any ideal of $R$, then $(B: M) M=B \cap M$ and $B M: M=B$. Since $L(R)$ is modular, this implies that $M$ is a principal element of $L(R)$ [2, Theorem 1].

The first step in proving our main result is to extend the result of Bogart and Johnson to rings which are not Noetherian. The proof of the following theorem was suggested by the proof of [6, Satz 1].

TheOREM 1. Let $R$ be a semilocal ring (not necessarily Noetherian). If $M$ is a principal element of $L(R)$ such that $0: M$ is contained in the Jacobson radical of $R$, then $M$ is a principal ideal of $R$.

Proof. Let $P_{1}, \cdots, P_{k}$ be the maximal ideals of $R$. For $i=1, \cdots$, $k, \quad\left(P_{i} M\right): M=0: M+P_{i} \subseteq P_{i}$, so that $P_{i} M \neq M$; choose $b_{i} \in M$, $b_{i} \notin P_{i} M$. Further, for $i=1, \cdots, k$, choose $a_{i} \in \prod_{j \neq i} P_{j}, a_{i} \notin P_{i}$. Set $a=\sum_{i=1}^{k} a_{i} b_{i}$. If, for some $i, a \in P_{i} M$, then $a_{i} b_{i} \in P_{i} M$; in this case, since $\left(a_{i}\right)+P_{i}=R$, we have $\left(b_{i}\right)=\left(a_{i} b_{i}\right)+P_{i} b_{i} \subseteq P_{i} M$. Thus, $a \notin P_{i} M$ for $i=1, \cdots, k$. Since $a \in M$ we have $(a)=((a): M) M$ and consequently $(a): M \Phi P_{i}$ for $i=1, \cdots, k$. Therefore, $(a): M=R$, i.e., $M=(a)$.

If $R$ has a single maximal ideal, then either $0: M$ is contained in that maximal ideal or $0: M=R$. Hence we have the

CoROllaRY. If $R$ is a local ring (not necessarily Noetherian), then every principal element of $L(R)$ is a principal ideal of $R$.

Theorem 2. An ideal $M$ of $R$ is a principal element of $L(R)$ if and only if $M$ is finitely generated and $M R_{P}$ is a principal ideal of $R_{P}$ for every maximal ideal $P$ of $R$.

Proof. Suppose that $M$ is finitely generated and $M R_{P}$ is principal for every maximal ideal $P$ of $R$. Then, for all ideals $A$ and $B$ of $R$, and for each such $P,(A \cap B: M) M R_{P}=(A M \cap B) R_{P}$. Hence $(A \cap B: M) M$ $=A M \cap B$. Likewise, the other required equality holds. Conversely, suppose that $M$ is a principal element of $L(R)$. By a result of Janowitz [7], $M$ is finitely generated. Let $P$ be a maximal ideal of $R$. Let $A^{\prime}$ and $B^{\prime}$ be ideals of $R_{P}$, and $A$ and $B$ their respective contractions to $R$. Then, 


$$
\begin{aligned}
\left(A^{\prime} \cap B^{\prime}: M R_{P}\right)\left(M R_{P}\right) & =(A \cap B: M) M R_{P}=(A M \cap B) R_{P} \\
& =A^{\prime}\left(M R_{P}\right) \cap B^{\prime},
\end{aligned}
$$

and the other required equality holds, showing that $M R_{P}$ is a principal element of $L\left(R_{P}\right)$. By the corollary to Theorem $1, M R_{P}$ is a principal ideal of $R_{P}$.

An ideal of $R$ is called regular if it contains an element which is not a zero divisor in $R$. We can use the connection between the invertible ideals of $R$ and the ideals of $R$ which are locally principal ideals $[4$, Teorema 1.1$]$ to obtain

CoRollary 1. A regular ideal of $R$ is a principal element of $L(R)$ if and only if it is invertible.

The next corollary contains [9, Theorem 2]; here "Prüfer ring" is used in the sense of Griffin [5].

CoRollary 2. A ring $R$ is a Prüfer ring if and only if every finitely generated regular ideal of $R$ is a principal element of $L(R)$.

Our final corollary is also an immediate consequence of the theorem of Dilworth mentioned earlier.

Corollary 3. If $R$ is Noetherian, and if $M$ is a principal element of $L(R)$, then every minimal prime divisor of $M$ has rank at most one.

The proof is the same as that of [4, Proposizione 1.2].

\section{REFERENCES}

1. K. P. Bogart, Structure theorems for regular local Noether lattices, Michigan Math. J. 15 (1968), 167-176. MR 37 \#2642.

2. - Distributive local Noether lattices, Michigan Math. J. 16 (1969), 215-223. MR $40 \# 5513$.

3. R. P. Dilworth, Abstract commutative ideal theory, Pacific J. Math. 12 (1962), 481-498. MR 26 \#1333.

4. S. Greco, Sugli ideali frazionari invertibili, Rend. Sem. Mat. Univ. Padova 36 (1966), 315-333. MR 34 \#1345.

5. M. Griffin, Prüfer rings with zero divisors, J. Reine Angew. Math. 239/240 (1969), 55-67. MR 41 \#1969.

6. A. Helms, Ein Beitrag zur algebraischen Geometrie, Math. Ann. 111 (1935), 438-458.

7. M. F. Janowitz, Principal multiplicative lattices, Pacific J. Math. 33 (1970), 653-656.

8. E. W. Johnson, A-transforms and Hilbertfunctions in local lattices, Trans. Amer. Math. Soc. 137 (1969), 125-139. MR 38 \#5675.

9. P. J. McCarthy, Arithmetical rings and multiplicative lattices, Ann. Mat. Pura Appl. (4) 82 (1969), 267-274. MR 40 \#1378.

UNIVERSITY OF KaNSAS, LAWRENCE, KaNSAS 66044 